\title{
A Candidate Gene Study for the Association of Host Single Nucleotide Polymorphisms with Liver Cirrhosis Risk in Chinese Hepatitis B Patients
}

\author{
Lijun Peng,,2 Jinsheng Guo,' Zhe Zhang,, Lili Liu,' Yirong Cao,, Hong Shi,' Jian Wang,, Jiyao Wang, \\ Scott L. Friedman, ${ }^{3}$ and John J. Sninsky ${ }^{4}$
}

Background and Aims: Recently, genetic association studies have linked a number of single nucleotide polymorphisms (SNPs) with liver fibrosis risk of hepatitis C. The present study was designed to validate the association of emerging SNPs with development of liver cirrhosis and chronicity in a Chinese population infected with hepatitis B virus (HBV). Methods: 714 Chinese subjects with persistent HBV infection (429 with evident liver cirrhosis and 285 without cirrhosis clinically or pathologically) and 280 subjects with spontaneous HBV clearance were studied. Six SNPs in five candidate genes were detected with the matrix-assisted laser desorption/ionization time-of-flight mass spectrometry (MALDI-TOF MS) method. The distribution of each polymorphism was compared between the age-matched cirrhotic and noncirrhotic subjects, and between subjects with persistent infection and spontaneous HBV clearance. Results: The rs2679757 polymorphism of antizyme inhibitor 1 (AZIN1) gene was associated with the risk of cirrhosis (odds ratio [OR] for GG + AG versus $\mathrm{AA}=1.47,95 \%$ confidence interval $[\mathrm{CI}]=1.08-2.01, p=0.01)$. So was rs886277 in the transient receptor potential cation channel subfamily $\mathrm{M}$, member 5 (TRPM5) gene ( $\mathrm{OR}$ for $\mathrm{CC}$ versus $\mathrm{CT}+\mathrm{TT}=1.63,95 \% \mathrm{CI}=1.20-2.22$, $p=0.002$ ). The frequencies of these two SNPs were also associated with the severity of decompensated cirrhosis based on the Child-Pugh classification. Genotype frequencies of other SNPs were not different between the cirrhotic and noncirrhotic groups. No SNPs were associated with the outcome of spontaneous HBV clearance. Conclusions: AZIN1 rs2679757 and TRPM5 rs886277 are associated with the risk of HBV-related liver cirrhosis in Chinese. The emerging SNPs warrant further clinical validation in other cohorts or ethnic groups, and could lead to mechanistic studies to reveal their contributions to fibrosis progression.

\section{Introduction}

$\mathbf{H}$ EPATITIS B VIRUs (HBV) infection is a public health threat worldwide, with up to two billion people harboring serological evidence of past or present infection, and 360 million with chronic infection (Shepard et al., 2006). HBV infection is endemic highly in China with an HBV surface antigen sero-positivity rate as high as 5\%-12\% (Chen et al., 2000). The natural history of HBV infection varies from spontaneous recovery postinfection, chronic asymptomatic carrier, to persistent infection with progression of fibrosis to decompensated cirrhosis and hepatocellular carcinoma, which confer a high mortality rate and heavy economic burden (Lavanchy, 2004; McMahon, 2009). Besides factors, such as alcohol abuse, gender, age, and viral genotypes contributing to this high variability (Powell et al., 2000; Fattovich et al., 2008), host genetic factors could be an important factor determining the outcome in hepatitis $\mathrm{B}$.

A single nucleotide polymorphism (SNP), the stable replacement of a single base in a human gene, is the most common genetic mutation in humans appearing in more than $1 \%$ of the population. A SNP may change the amino acid sequence and subsequently the protein function, or alter the gene expression in the level of RNA transcription, splicing, or protein translation based on its location in the genome. Although it is uncommon that a SNP is identified as a diseasecausing variant, genetic variation may impact significantly on the disease susceptibility. To date, genome-wide association studies (GWAS) and candidate gene association studies have been performed abundantly to delineate the genetic impact of

\footnotetext{
${ }^{1}$ Division of Digestive Diseases, Department of Internal Medicine, Zhong Shan Hospital, Shanghai Medical College, Fu Dan University, Shanghai, China.

${ }^{2}$ Department of Gastroenterology, Linyi People's Hospital, Linyi, Shandong, China.

${ }^{3}$ Division of Liver Diseases, Mount Sinai Hospital, Icahn School of Medicine at Mount Sinai, New York, New York.

${ }^{4}$ Department of Discovery Research, Celera Corporation, Alameda, California.
} 
SNPs on human liver diseases (Day, 2005; Karlsen et al., 2010). Notebly, Huang et al. (2007) identified 7 SNPs residing in seven genes were associated with liver fibrosis in Caucasian patients infected with hepatitis $\mathrm{C}$ virus $(\mathrm{HCV})$. The seven genes, including toll-like receptor 4 (TLR4), degenerative spermatocyte homolog 1, lipid desaturase (DEGS1), syntaxinbinding protein 5-like (STXBP5L), antizyme inhibitor 1 (AZIN1), transient receptor potential cation channel subfamily M, member 5 (TRPM5), adaptor-related protein complex 3 , sigma 2 subunit (AP3S2), and aquaporin 2 (AQP2) may be functional in the pathogenesis of liver fibrosis. Cirrhosis risk scores (CRS) built on the seven SNPs were predictive for fibrosis progression in chronic hepatitis $\mathrm{C}$ patients in longitudinal studies (Marcolongo et al., 2009; Curto et al., 2011). In the studies on nonalcoholic fatty liver disease and alcoholic liver disease, rs738409 in patatin-like phospholipase domain containing 3 (PNPLA3) has been strongly associated with the disease' susceptibility, severity and clinical outcomes (Romeo et al., 2008; Tian et al., 2009; Valenti et al., 2010). Although several polymorphisms in genes of interferon-gamma (IFN- $\gamma$ ), tumor necrosis factor-alpha (TNF- $\alpha$ ), chemokine receptor 5 (CCR5), and HLA-DP were reported to be associated with persistent HBV infection (Cheong et al., 2006; Du et al., 2006; Liu et al., 2006; Suneetha et al., 2006; Kamatani et al., 2009), few studies have investigated the roles of host gene polymorphisms in development of HBV-related liver cirrhosis.

As hepatitis $B$ has similar fibrogenic process as hepatitis $C$ or other etiologies of liver diseases once the liver injuries started, they may share common genetic risk factors for fibrosis progression. Therefore, the present study was designed to evaluate the association of cirrhosis risk SNPs reported in Caucasian hepatitis $C$ patients with the risk of cirrhosis and the likelihood of chronicity in Chinese subjects infected with HBV.

\section{Materials and Methods}

\section{Study population}

A total of 994 participants were enrolled for this casecontrol study in Zhong Shan Hospital, Fu Dan University between March 2009 and January 2011. They were all Chinese Han subjects from an epidemic region of HBV in the Eastern coastal region of China. The study was approved by the ethical committee of the hospital and was registered on the Chinese Clinical Trials Register (www.chictr.org) numbered ChiCTRONC-00000439. The protocol conformed to the provisions of the Declaration of Helsinki (as revised in Tokyo, 2004). Informed consent was obtained from all subjects.

Information, including hepatitis B history, alcohol consumption, serum HBV markers, liver function test, prothrombin time, platelets count, liver imaging (computed tomography or magnetic resonance imaging), and liver histology if available, was collected for the diagnosis and judging the severity of HBV-related liver diseases. Alcohol doses of $>40 \mathrm{~g} /$ day in males and $>20 \mathrm{~g} /$ day in females for at least 5 years were deemed alcoholics and excluded from the study. Serum HBV markers, including hepatitis B surface antigen (HBsAg), antibody to HBsAg (anti-HBs), hepatitis B e antigen (HBeAg), antibody to HBeAg (anti-HBe), and antibody to hepatitis B core antigen (anti-HBc) of IgG type were measured with chemiluminescent assays (Roche Diagnostics). The copy numbers of plasma HBV-DNA were measured with Roche Lightcycler ${ }^{\circledR}$ system. Child-Pugh scores were calculated for patients with advanced liver disease. Patients with evidence of chronic hepatitis $\mathrm{C}$, autoimmune liver diseases, nonalcoholic steatohepatitis, drug-induced liver disease, Wilson's disease, and serious clinical condition of other organs were excluded. The diagnosis of these diseases was referred to current clinical practice guidelines (http://aasld.org/practice guidelines/pages/guidelinelisting.aspx). Patients with previous anti-HBV therapy history were also excluded. Patients with hepatocellular carcinoma, which is one of the major complications of hepatitis B, were enrolled in the case group if they were cirrhotic.

Clinical phenotypes were grouped as persistent infection with evident liver cirrhosis (cirrhotic group) or persistent infection without liver cirrhosis (noncirrhotic group). HBV infection was defined as HBsAg positive for more than 6 months, with anti-HBs negative and anti-HBc positive. Persistent infection denoted an infection status for at least 10 years according to disease's history. Spontaneous HBV clearance was defined as HBsAg negative, HBeAg negative, anti-HBc positive, anti-HBs positive, and HBV-DNA negative in healthy population. Cirrhosis was determined based on the clinical manifestations of decompensated cirrhosis (e.g., ascites, varices hemorrhage, hepatic encephalopathy, spontaneous bacterial peritonitis), typical radiological findings of cirrhosis and portal hypertension (e.g., hepatatrophia, cirrhotic nodules, ascites, varices, portal vein dilation, splenomegaly), cirrhotic pathology in liver biopsy (with the highest fibrosis stage of METAVIR 4), and laboratory features pertaining to portal hypertension and decompensated liver function (e.g., low platelets count, low white blood cell count, hyperbilirubinemia, hypoalbuminemia, prothrombin time prolongation). The cirrhotic group of patients was subgrouped into Child-Pugh A group, and Child-Pugh B plus C group based on the classification. The noncirrhotic group included mainly inactive HBsAg carriers with persistently normal alanine aminotransferase level, positive anti-HBe, and undetectable HBV-DNA in two blood tests within over a-year duration, and without any of the above cirrhosis signs.

\section{Selection of polymorphisms}

Six SNPs within five genes as reported by previous studies to be associated with cirrhosis risk were selected. Their analysis was performed using a single Multiplex PCR amplification system. Details of the SNP location (missense, synonymous, promoter, intronic or other changes), known function or potential biological impact of the SNPs are listed in Table 1. (Ternes et al., 2002; Beutler, 2004; Mangold, 2006; Takata, 2006; Huang et al., 2007; Kaske et al., 2007; Kraveka et al., 2007; Seki et al., 2007; Guo et al., 2009).

\section{Preparation of DNA samples}

From each subject, $2 \mathrm{~mL}$ blood was collected in an EDTAcontaining tube. Genomic DNA was extracted with QIAamp DNA Blood Midi Kit (Qiagen). DNA samples were analyzed by nucleic acid spectrophotometer and gel electrophoresis to ensure the purity and for quantification.

\section{SNPs detection}

SNPs were genotyped using matrix-assisted laser desorption/ionization time-of-flight mass spectrometry (MALDI- 
Table 1. General Information on the Six Candidate SNPs in the Study

\begin{tabular}{|c|c|c|c|c|c|}
\hline $\begin{array}{l}\text { Rs } \\
\text { number }\end{array}$ & Gene & $\begin{array}{c}\text { SNP } \\
\text { location }\end{array}$ & Reported clinical association & Biological function of gene & $\begin{array}{l}\text { Functional } \\
\text { linkage with } \\
\text { fibrogenesis }\end{array}$ \\
\hline rs2878771 & AQP2 & UTR & HCV-related liver fibrosis & Water reabsorption, vasopressin regulation & Unknown \\
\hline rs2679757 & AZIN1 & Intron region & HCV-related liver fibrosis & Polyamine biosynthesis, cell proliferation & Unknown \\
\hline rs4290029 & DEGS1 & Intergenic & HCV-related liver fibrosis & $\begin{array}{l}\text { Lipid metabolism and transport, } \\
\text { cell growth }\end{array}$ & Unknown \\
\hline rs960312 & TLR4 & Intergenic & HCV-related liver fibrosis & $\begin{array}{l}\text { LPS-stimulated inflammatory } \\
\text { responses, profibrogenic signals }\end{array}$ & Unknown \\
\hline rs1927911 & TLR4 & Intron region & HCV-related liver fibrosis & $\begin{array}{l}\text { LPS-stimulated inflammatory } \\
\text { responses, profibrogenic signals }\end{array}$ & Unknown \\
\hline rs886277 & TRPM5 & Missense & HCV-related liver fibrosis & Taste responses & Unknown \\
\hline
\end{tabular}

Clinical association and biological function of genes are based on the references shown in the text.

SNP, single nucleotide polymorphism; AQP2, aquaporin 2; UTR, untranslated region; HCV, hepatitis C virus; AZIN1, antizyme inhibitor 1; DEGS1, degenerative spermatocyte homolog 1, lipid desaturase; TLR4, toll-like receptor 4; LPS, lipopolysaccharides; TRPM5, transient receptor potential cation channel subfamily $\mathrm{M}$, member 5 .

TOF MS) (Jurinke et al., 2004; Ragoussis et al., 2006), which determines the allele-specific primer extension products with Sequenom's MassARRAY system and iPLEX technology (Sequenom; www.sequenom.com). Briefly, Multiplex PCR amplifications of amplicons containing SNPs of interest were performed using Sequenom HotStart Taq Polymerase on an Applied Biosystems 9700 Real-Time PCR instrument (Applied Biosystems) with $10 \mathrm{ng}$ genomic DNA of each sample. Enzymatic single-base primer extension reactions were performed to add four mass-modified nucleotides, that is, $2^{\prime}$, 3'-dideoxynucleoside 5'-triphosphate (ddNTP), into the polymorphic site, which allow the production of allelespecific extension products of different mass. The iPLEX reaction products were loaded onto 384-spot SpectroChips (Sequenom) and further analysed by MassARRAY Analyzer Compact System (Sequenom). After laser desorption/ionization, automated spectral acquisition analysis was performed and the spectral data was saved to the MassARRAY database.

\section{Statistical analysis}

The parametric Student $t$-test and the nonparametric Wilcoxon rank-sum test were utilized to assess differences in the mean data between groups for the characteristics and liver function profiles of the studied populations. The frequency of genotypes and alleles was determined by direct gene counting method. Pearson $\chi^{2}$ test or Fisher's exact probability test was used for univariate analysis of the allele distribution in difference groups. The $\chi^{2}$ test was also used in the evaluation of Hardy-Weinberg equilibrium. Binary logistic regression analysis was performed to determine the adjusted significance, where age and sex were covariables. The genotypic distributions of each SNP between different phenotypic groups were analyzed. Each statistically significant SNP in univariate analysis was further assessed with logistic regression analysis using the dominant, recessive and additive genetic models, and the odds ratio (OR) and 95\% confidence interval (CI) were calculated. Furthermore, the association of the significant SNPs with the severity of cirrhosis, which was judged by Child-Pugh classification, was analyzed. Statistics was performed with SPSS version 15.0 software (SPSS). A $p$-value $<0.05$ was considered to be statistically significant.

\section{Results}

\section{Population characteristics}

The characteristics of the 994 subjects in the study are shown in Table 2. There was no difference between the

Table 2. Characteristics of the Study Population

\begin{tabular}{|c|c|c|c|c|c|}
\hline \multirow[b]{2}{*}{ Parameters } & \multicolumn{2}{|c|}{ Persistent infection (c) } & \multirow[b]{2}{*}{ Spontaneous clearance $(d)$} & \multicolumn{2}{|c|}{$\mathrm{p}$-Value } \\
\hline & Cirrhosis (a) & Noncirrhosis $(b)$ & & $a, b$ & $c, d$ \\
\hline Total $n=994$ & 429 & 285 & 280 & - & - \\
\hline Age (year) & $49.3 \pm 10.5$ & $47.8 \pm 13.4$ & $48.1 \pm 10.1$ & 0.12 & 0.73 \\
\hline Male, $n(\%)$ & 334 (77.9) & 205 (71.9) & $211(75.4)$ & 0.07 & 0.97 \\
\hline Platelet $\left(10^{9} / \mathrm{L}\right)$ & $96.1 \pm 59.6$ & $195.3 \pm 62.1$ & $210.9 \pm 64.9$ & $<0.01$ & $<0.01$ \\
\hline Total bilirubin $(\mu \mathrm{M})$ & $33.1 \pm 64.2$ & $12.9 \pm 11.0$ & $11.2 \pm 4.5$ & $<0.01$ & $<0.01$ \\
\hline $\operatorname{ALT}(\mathrm{IU} / \mathrm{L})$ & $77.4 \pm 169.0$ & $30.1 \pm 15.4$ & $22.4 \pm 11.9$ & $<0.01$ & $<0.01$ \\
\hline Albumin (g/L) & $35.1 \pm 6.8$ & $41.1 \pm 4.2$ & $40.4 \pm 3.6$ & $<0.01$ & $<0.01$ \\
\hline PT (second) & $14.0 \pm 3.4$ & $11.5 \pm 0.90$ & $11.7 \pm 0.73$ & $<0.01$ & $<0.01$ \\
\hline Child-Pugh grades, $n$ & A:235, B:145, C:49 & A:285, B:0, C:0 & A:280, B:0, C:0 & $<0.01$ & $<0.01$ \\
\hline
\end{tabular}

Normal numeral ranges for platelet, total bilirubin, ALT, albumin, and PT were 101 to $320\left(10^{9} / \mathrm{L}\right), 3.4$ to 20.4 ( $\left.\mu \mathrm{M}\right),<75$ (IU/L), 35 to 52 (g/L), and 10 to $13(\mathrm{~s})$, respectively.

Data were expressed as mean \pm standard deviation. Letters $a, b, c$, and d denote data of cirrhosis, noncirrhosis, persistent infection, and spontaneous clearance groups, respectively.

$\mathrm{ALT}$, alanine aminotransferase; PT, prothrombin time. 
Table 3. Minor Allele Frequency of Genotyped SNPs

\begin{tabular}{llcc}
\hline SNP & Allele & $\begin{array}{c}\text { Detected MAF } \\
\text { in this study }\end{array}$ & $\begin{array}{c}\text { Reported MAF } \\
\text { in Caucasians }\end{array}$ \\
\hline AQP2 rs2878771 & G/C & C: $40.9 \%$ & C: $18.6 \%$ \\
AZIN1 rs2679757 & A/G & G: $22.9 \%$ & G: $40.8 \%$ \\
DEGS1 rs4290029 & G/C & C: $39.9 \%$ & C: $22.4 \%$ \\
TLR4 rs960312 & A/G & G: $27.6 \%$ & G: $15.8 \%$ \\
TLR4 rs1927911 & C/T & T: $41.5 \%$ & T: $26.5 \%$ \\
TRPM5 rs886277 & C/T & T: $34.7 \%$ & C: $34.8 \%$ \\
\hline
\end{tabular}

MAF in Caucasians are based on the the SNP database of the national center for biotechnology information.

MAF, minor allele frequency.

cirrhotic and noncirrhotic groups in mean age $(p=0.12)$. There is a little higher ratio of male gender in the cirrhotic group when compared with the noncirrhotic group $(77.9 \%$ vs. $71.9 \%$, $p=0.07)$. There were no significant differences between persistent infection group and spontaneous clearance group in mean age and sex ratio ( $p>0.05)$. The blood tests and ChildPugh grades suggested apparent liver decompensation in the cirrhotic group $(p<0.01)$.

\section{Association of the SNPS with liver cirrhosis and persistent infection}

Minor allele frequency of each SNP is shown in Table 3. The allele frequencies of these SNPs corresponded well with those reported in the SNP database of the national center for biotechnology information. The frequencies were different between Chinese and Caucasians.

The genotypic distributions were in Hardy-Weinberg equilibrium except TLR4 rs960312 in this population (Table 4). All SNPs showed no significant association with spontaneous

Table 4. Genotypic Frequency for Each SNP in Persistent Infection Group and Spontaneous Clearance Group

\begin{tabular}{lcrrrr}
\hline $\begin{array}{l}\text { Gene } \\
\text { and SNP }\end{array}$ & Genotype & $\begin{array}{c}\text { Persistent } \\
\text { infection, } \\
\mathrm{n}(\%)\end{array}$ & $\begin{array}{c}\text { Spontaneous } \\
\text { clearance, } \\
\mathrm{n}(\%)\end{array}$ & $\begin{array}{c}\mathrm{p} \text { - } \\
\text { Value }\end{array}$ & HWP \\
\hline AQP2 & GG & $243(34.2)$ & $97(34.9)$ & 0.91 & 0.54 \\
rs2878771 & GC & $349(49.2)$ & $138(49.6)$ & & \\
AZIN1 & CC & $118(16.6)$ & $43(15.5)$ & & \\
rs2679757 & AA & $411(57.8)$ & $170(61.4)$ & 0.57 & 0.23 \\
& GG & $266(37.4)$ & $96(34.7)$ & & \\
DEGS1 & CC & $107(15.0)$ & $57(20.6)$ & 0.10 & 0.38 \\
rs4290029 & CG & $340(47.8)$ & $121(43.7)$ & & \\
TLR4 & GG & $265(37.2)$ & $99(35.7)$ & & \\
rs960312 & AA & $379(53.2)$ & $158(56.8)$ & 0.43 & 0.003 \\
& GG & $267(37.5)$ & $92(33.1)$ & & \\
TLR4 & GG & $66(9.3)$ & $28(10.1)$ & & \\
rs1927911 & TC & $125(17.6)$ & $51(18.3)$ & 0.86 & 0.46 \\
& CC & $251(35.1)$ & $134(48.2)$ & & \\
TRPM5 & CC & $310(43.5)$ & $113(40.8)$ & 0.65 & 0.88 \\
rs886277 & CT & $319(44.8)$ & $127(45.8)$ & & \\
& TT & $83(11.7)$ & $37(13.4)$ & &
\end{tabular}

HWP, $p$-value for Hardy-Weinberg equilibrium test.
HBV clearance (Table 4). For cirrhosis-related analysis, rs2679757 in AZIN1 gene and rs886277 in TRPM5 gene both showed a significant association with the development of liver cirrhosis ( $p=0.003$ and 0.001 , respectively) (Table 5). Regression analysis with adjustment for age and sex revealed that rs2679757 genotypes were associated with a significant risk of liver cirrhosis with the use of either a dominant $(\mathrm{OR}=1.47,95 \% \mathrm{CI}: 1.08-2.01, p=0.01)$ or an additive genetic model (OR $=1.43,95 \%$ CI: $1.04-1.96, p=0.03)$ for the $\mathrm{G}$ risk allele (Table 6). Similarly, rs886277 genotypes in TRPM5 gene were associated with liver cirrhosis risk in the recessive model for the risk $\mathrm{C}$ allele $(\mathrm{OR}=1.63,95 \% \mathrm{CI}: 1.20-2.22, p=0.002)$ (Table 6). In a further analysis for the association of rs 2679757 and rs886277 polymorphisms with the severity of cirrhosis judged by Child-Pugh classification, GG homozygote and AG heterozygote (GG+AG) of AZIN1 rs2679757 as a risk factor was found to have a higher frequency in Child-Pugh B plus C subgroup than in Child-Pugh A subgroup $\left(\chi^{2}=8.54\right.$, $p=0.003)$. The analysis of CC homozygote of TRPM5 rs886277 as another risk factor also achieved a similar result $\left(\chi^{2}=8.4\right.$, $p=0.004)$.

\section{Discussion}

In the present study, six candidate SNPs in five genes were evaluated for their association with the risk of liver cirrhosis and the outcome of chronicity in a Chinese population infected with HBV. We defined the cases and controls primarily by clinical evidence. As the disease progression thereafter could not be anticipated for healthy HBV carriers at the study time, we enrolled participants with a similar mean age for the case and control groups. We found rs2679757 polymorphism in the AZIN1 gene and rs886277 in the TRPM5 gene were associated with a significant risk of HBV-related liver cirrhosis. None of these cirrhosis-related SNPs were associated with HBV clearance, which may indicate that the chronicity of $\mathrm{HBV}$ infection and fibrosis progression are two distinct

Table 5. Genotypic Frequency for Each SNP in the Cirrhotic AND Noncirrhotic Group

\begin{tabular}{|c|c|c|c|c|c|}
\hline $\begin{array}{l}\text { Gene } \\
\text { and SNP }\end{array}$ & Genotype & $\begin{array}{c}\text { Cirrhotic } \\
\text { group, } \\
\text { n }(\%)\end{array}$ & $\begin{array}{c}\text { Noncirrhotic } \\
\text { group, } \\
\mathrm{n}(\%)\end{array}$ & $\begin{array}{c}\mathrm{p}^{-} \\
\text {Value }\end{array}$ & HWP \\
\hline \multirow{3}{*}{$\begin{array}{l}\text { AQP2 } \\
\text { rs2878771 }\end{array}$} & GG & 143 (33.6) & $100(35.2)$ & \multirow[t]{3}{*}{0.86} & \multirow[t]{3}{*}{0.70} \\
\hline & GC & $210(49.3)$ & $139(48.9)$ & & \\
\hline & $\mathrm{CC}$ & 73 (17.1) & 45 (15.8) & & \\
\hline \multirow{3}{*}{$\begin{array}{l}\text { AZIN1 } \\
\quad \text { rs2679757 }\end{array}$} & $\mathrm{AA}$ & $231(54.1)$ & $180(63.4)$ & \multirow[t]{3}{*}{0.003} & \multirow[t]{3}{*}{0.28} \\
\hline & AG & $172(40.3)$ & $94(33.1)$ & & \\
\hline & GG & 24 & 10( & & \\
\hline \multirow{3}{*}{$\begin{array}{l}\text { DEGS1 } \\
\quad \text { rs4290029 }\end{array}$} & $\mathrm{CC}$ & 62 & 45 & \multirow[t]{3}{*}{0.80} & \multirow[t]{3}{*}{0.90} \\
\hline & CG & 208 & 132 & & \\
\hline & & ) & 108 & & \\
\hline \multirow{3}{*}{$\begin{array}{l}\text { TLR4 } \\
\text { rs960312 }\end{array}$} & AA & $232(54.2)$ & 147 (51.8) & \multirow[t]{3}{*}{0.79} & \multirow[t]{3}{*}{0.06} \\
\hline & $\mathrm{AG}$ & $158(36.9)$ & 109 (38.4) & & \\
\hline & GG & $38(8.9)$ & $28(9.9)$ & & \\
\hline \multirow{3}{*}{$\begin{array}{l}\text { TLR4 } \\
\quad \text { rs1927911 }\end{array}$} & $\mathrm{TT}$ & 70 (16.3) & 55 (19.5) & \multirow[t]{3}{*}{0.55} & \multirow[t]{3}{*}{0.47} \\
\hline & TC & $205(47.8)$ & $130(46.1)$ & & \\
\hline & $\mathrm{CC}$ & 154 (35.9) & $97(34.4)$ & & \\
\hline \multirow{3}{*}{$\begin{array}{l}\text { TRPM5 } \\
\text { rs886277 }\end{array}$} & $\mathrm{CC}$ & $206(48.1)$ & $104(36.6)$ & \multirow[t]{3}{*}{0.001} & \multirow[t]{3}{*}{0.94} \\
\hline & $\mathrm{CT}$ & $176(41.1)$ & $143(50.4)$ & & \\
\hline & $\mathrm{TT}$ & $46(10.7)$ & $37(13.0)$ & & \\
\hline
\end{tabular}


Table 6. Age- And Sex-Adjusted Odds Ratio for AZIN1 Rs2679757 And TRPM5 Rs886277 GenotyPes in the Cirrhotic Group Versus the Noncirrhotic Group

\begin{tabular}{|c|c|c|c|c|c|}
\hline$S N P$ & Comparison & OR & $95 \% C I$ & $\chi^{2}$ & $\mathrm{p}$-Value \\
\hline \multirow[t]{4}{*}{ AZIN1 rs2679757 } & G vs. A & 1.38 & $1.07-1.79$ & 6.15 & 0.01 \\
\hline & Dominant model GG + AG vs. AA & 1.47 & $1.08-2.01$ & 6.03 & 0.01 \\
\hline & Recessive model GG vs. AG + AA & 1.67 & $0.78-3.55$ & 1.65 & 0.19 \\
\hline & Additive model AG vs. AA & 1.43 & $1.04-1.96$ & 4.79 & 0.03 \\
\hline \multirow[t]{4}{*}{ TRPM5 rs886277 } & C vs. T & 1.36 & $1.09-1.69$ & 7.23 & 0.01 \\
\hline & Dominant model CC $+\mathrm{CT}$ vs. TT & 1.27 & $0.80-2.03$ & 0.86 & 0.31 \\
\hline & Recessive model CC vs. CT + TT & 1.63 & $1.20-2.22$ & 9.20 & 0.002 \\
\hline & Additive mode CT vs. TT & 0.98 & $0.60-1.60$ & 0.002 & 0.93 \\
\hline
\end{tabular}

OR, odds ratio; $\mathrm{CI}$, confidence interval.

processes that are attributable to different factors associated with different genetic determinants.

This study provides the first evidence that the rs2679757 and rs886277 were associated with the development and severity of cirrhosis in hepatitis B patients. For rs2679757 in AZIN1 gene, the $G$ allele is the risk factor for cirrhosis. Functionally, AZIN1 stabilizes ornithine decarboxylase (ODC) by blocking the function of antizyme, which suppresses ODC activity and accelerates its proteasomal degradation (Mangold, 2006). ODC is a key enzyme in polyamine biosynthesis induced by various growth and differentiation stimuli. The observed association suggests a possible role of AZIN1-related activity in hepatic pathology and fibrogenesis. It was reported that another AZIN1 polymorphism, rs62522600 was associated with development of cirrhosis in Caucasian hepatitis C patients (Huang et al., 2007; Paris et al., 2011). However, this locus was not shown with diverse genotypes in Chinese. The polymorphic nature of the AZIN1 gene may modulate individual responses to liver damage and serve as a common functional SNP in HBVand HCV-induced hepatic fibrogenesis. The presence of this SNP in the intronic region of the AZIN1 gene may alter the gene expression and function by creating an alternative splice site and thus affects post-transcriptional regulation of the gene. TRPM5 is one member of the melastatin subfamily of transient receptor potential ion channels that are widely distributed in mammalian tissues (Prawitt et al., 2003). It is proved to be a calcium-activated cation channel that functions in taste signal transduction (Kaske et al., 2007). The specific physiological function of TRPM5 in liver is so far unknown. The variant rs 886277 leading to a missense change in amino acid sequence may influence the biological function of the TRPM5 protein. Further research is encouraged to validate these emerging risk SNPs in other hepatitis B population or ethnic groups, and to reveal the mechanism links between these polymorphisms and liver fibrosis.

There was no association of other SNPs with cirrhosis risk. The discrimination between our study and previous reports may be due to the following reasons: (1) This is a crosssectional study, which may not as accurate as the longitudinal study in the evaluation of the disease occurrence or progression, (2) The candidate genes and SNPs were selected based on the studies on different kinds of viral hepatitis; thus, specific pathogenetic differences among different liver diseases may account for the divergent results, (3) Ethnic differences of allelic frequencies and diseases susceptibility may exist between Caucasians and Chinese.
As the present study was mainly focused on host genetic factors, we didn't test the HBV genotypes and gene polymorphisms of the virus. The participants enrolled in this study are from a centralized area of Chinese mainland where HBV genotypes B and C are most prevalent. The impact of the HBV genotypes $B$ and $C$ on the long-term prognosis of chronic hepatitis B, such as cirrhosis, cirrhosis-related complications, and/or hepatocellular carcinoma is controversial (Yuen et al., 2003; Yin et al., 2010). Further analysis for the association between the risk SNPs and HBV genotypes in larger cohorts is warranted to delineate genotype-specific mechanisms in relation to fibrosis progression.

In this study, we developed a reliable medium-throughput genotyping platform using the Sequenom MassARRAY system for testing gene variants. This methodology allows simultaneous determination of multiple SNPs in one set with hundreds of samples (Jurinke et al., 2004; Ragoussis et al., 2006), and has potential to generate convenient polygenic tests useful in clinical setting.

In conclusion, two polymorphisms, rs2679757 in AZIN1 gene and rs886277 in TRPM5 gene, were verified to be associated with liver cirrhosis risk in Chinese hepatitis B patients. The emerging SNPs related to cirrhosis warrant further basic investigation and clinical validation in hepatitis B. Larger efforts, including GWAS are needed for generating CRS signatures specifically for Chinese hepatitis $\mathrm{B}$ patients. These genetic signatures may allow better determination of prognosis, offer opportunities for prevention, and identify new therapeutic targets for the treatment of liver cirrhosis.

\section{Acknowledgments}

This work was supported by Shanghai Pujiang Talent Program 2009 (09PJ1402600) to Jinsheng Guo, and Wang BaoEn Liver Fibrosis Research Foundation (20090001) to Jiyao Wang.

\section{Author Disclosure Statement}

No competing financial interests exist.

\section{References}

Beutler B (2004) Inferences, questions and possibilities in Tolllike receptor signalling. Nature 430:257-263.

Chen CJ, Wang LY, Yu MW (2000) Epidemiology of hepatitis B virus infection in the Asia-Pacific region. J Gastroenterol Hepatol 15 Suppl:E3-E6. 
Cheong JY, Cho SW, Hwang IL, et al. (2006) Association between chronic hepatitis B virus infection and interleukin-10, tumor necrosis factor-alpha gene promoter polymorphisms. J Gastroenterol Hepatol 21:1163-1169.

Curto TM, Lagier RJ, Lok AS, et al. (2011) Predicting cirrhosis and clinical outcomes in patients with advanced chronic hepatitis $\mathrm{C}$ with a panel of genetic markers (CRS7). Pharmacogenet Genomics 21:851-860.

Day CP (2005) Genetic studies to identify hepatic fibrosis genes and SNPs in human populations. Methods Mol Med 117: 315-331.

Du T, Guo XH, Zhu XL, et al. (2006) Association of TNF-alpha promoter polymorphisms with the outcomes of hepatitis B virus infection in Chinese Han population. J Viral Hepat 13:618-624.

Fattovich G, Bortolotti F, Donato F (2008) Natural history of chronic hepatitis B: special emphasis on disease progression and prognostic factors. J Hepatol 48:335-352.

Guo J, Loke J, Zheng F, et al. (2009) Functional linkage of cirrhosis-predictive single nucleotide polymorphisms of Tolllike receptor 4 to hepatic stellate cell responses. Hepatology 49:960-968.

Huang H, Shiffman ML, Friedman S, et al. (2007) A 7 gene signature identifies the risk of developing cirrhosis in patients with chronic hepatitis C. Hepatology 46:297-306.

Jurinke C, Oeth P, van den Boom D (2004) MALDI-TOF mass spectrometry: a versatile tool for high-performance DNA analysis. Mol Biotechnol 26:147-164.

Kamatani Y, Wattanapokayakit S, Ochi H, et al. (2009) A genomewide association study identifies variants in the HLA-DP locus associated with chronic hepatitis B in Asians. Nat Genet 41: 591-595.

Karlsen TH, Melum E, Franke A (2010) The utility of genome-wide association studies in hepatology. Hepatology 51:1833-1842.

Kaske S, Krasteva G, Konig P, et al. (2007) TRPM5, a taste-signaling transient receptor potential ion-channel, is a ubiquitous signaling component in chemosensory cells. BMC Neurosci 8:49.

Kraveka JM, Li L, Szulc ZM, et al. (2007) Involvement of dihydroceramide desaturase in cell cycle progression in human neuroblastoma cells. J Biol Chem 282:16718-16728.

Lavanchy D (2004) Hepatitis B virus epidemiology, disease burden, treatment, and current and emerging prevention and control measures. J Viral Hepat 11:97-107.

Liu M, Cao B, Zhang H, et al. (2006) Association of interferongamma gene haplotype in the Chinese population with hepatitis B virus infection. Immunogenetics 58:859-864.

Mangold U (2006) Antizyme inhibitor: mysterious modulator of cell proliferation. Cell Mol Life Sci 63:2095-2101.

Marcolongo M, Young B, Pero FD, et al. (2009) A seven-gene signature (cirrhosis risk score) predicts liver fibrosis progression in patients with initially mild chronic hepatitis C. Hepatology 50:1038-1044.

McMahon BJ (2009) The natural history of chronic hepatitis B virus infection. Hepatology 49:S45-S55.

Paris AJ, Snapir Z, Christopherson CD, et al. (2011) A polymorphism that delays fibrosis in hepatitis $C$ promotes alternative splicing of AZIN1, reducing fibrogenesis. Hepatology 54:2198-2207.

Powell EE, Edwards-Smith CJ, Hay JL, et al. (2000) Host genetic factors influence disease progression in chronic hepatitis $\mathrm{C}$. Hepatology 31:828-833.

Prawitt D, Monteilh-Zoller MK, Brixel L, et al. (2003) TRPM5 is a transient $\mathrm{Ca} 2+$-activated cation channel responding to rapid changes in $[\mathrm{Ca} 2+]$ i. Proc Natl Acad Sci U S A 100:1516615171.

Ragoussis J, Elvidge GP, Kaur K, et al. (2006) Matrix-assisted laser desorption/ionisation, time-of-flight mass spectrometry in genomics research. PLoS Genet 2:e100.

Romeo S, Kozlitina J, Xing C, et al. (2008) Genetic variation in PNPLA3 confers susceptibility to nonalcoholic fatty liver disease. Nat Genet 40:1461-1465.

Seki E, De Minicis S, Osterreicher CH, et al. (2007) TLR4 enhances TGF-beta signaling and hepatic fibrosis. Nat Med 13:1324-1332.

Shepard CW, Simard EP, Finelli L, et al. (2006) Hepatitis B virus infection: epidemiology and vaccination. Epidemiol Rev 28:112-125.

Suneetha PV, Sarin SK, Goyal A, et al. (2006) Association between vitamin D receptor, CCR5, TNF-alpha and TNF-beta gene polymorphisms and HBV infection and severity of liver disease. J Hepatol 44:856-863.

Takata K (2006) Aquaporin-2 (AQP2): its intracellular compartment and trafficking. Cell Mol Biol (Noisy-le-grand) 52: 34-39.

Ternes P, Franke S, Zahringer U, et al. (2002) Identification and characterization of a sphingolipid delta 4-desaturase family. J Biol Chem 277:25512-25518.

Tian C, Stokowski RP, Kershenobich D, et al. (2009) Variant in PNPLA3 is associated with alcoholic liver disease. Nat Genet 42:21-23.

Valenti L, Al-Serri A, Daly AK, et al. (2010) Homozygosity for the patatin-like phospholipase-3/adiponutrin I148M polymorphism influences liver fibrosis in patients with nonalcoholic fatty liver disease. Hepatology 51:1209-1217.

Yin JH, Zhao J, Zhang HW, et al. (2010) HBV genotype C is independently associated with cirrhosis in community-based population. World J Gastroenterol 16:379-383.

Yuen MF, Sablon E, Yuan HJ, et al. (2003) Significance of hepatitis $\mathrm{B}$ genotype in acute exacerbation, $\mathrm{HBeAg}$ seroconversion, cirrhosis-related complications, and hepatocellular carcinoma. Hepatology 37:562-567.

Address correspondence to: Jinsheng Guo, MD Division of Digestive Diseases Department of Internal Medicine Zhong Shan Hospital Shanghai Medical College Fu Dan University 180 Feng Lin Road Shanghai 200032 China

E-mail: guo.jinsheng@zs-hospital.sh.cn

Jiyao Wang, MD Division of Digestive Diseases Department of Internal Medicine Zhong Shan Hospital Shanghai Medical College Fu Dan University 180 Feng Lin Road Shanghai 200032 China

E-mail: wang.jiyao@zs-hospital.sh.cn 